\title{
Energy expenditure of walking for adult patients with spinal cord lesions using the reciprocating gait orthosis and functional electrical stimulation
}

\author{
L Sykes $^{1}$, IG Campbell ${ }^{2}$, ES Powell ${ }^{1}$, ERS Ross ${ }^{1}$ and J Edwards ${ }^{3}$ \\ ${ }^{1}$ North Western Orthotic Unit, Clinical Sciences Building, Hope Hospital, Eccles Old Road, Salford M6 8HD; \\ ${ }^{2}$ Division of Sports Science, the Manchester Metropolitan University, Crewe and Alsager Faculty, Hassall Road, \\ Alsager, Stoke on Trent ST7 2HL; ${ }^{3}$ Spinal Injuries Centre, District General Hospital, Town Lane, Kew, Southport, \\ UK PR8 $6 \mathrm{NJ}$
}

\begin{abstract}
A major factor influencing compliance with walking orthoses following spinal cord damage, is the energy requirement associated with them. We compared ambulatory energy expenditure in subjects using the reciprocating gait orthosis (RGO) with and without functional electrical stimulation (FES) of the thigh muscles at self selected walking speeds. Five adult subjects (median age 34 years, range $24-37$ ) with spinal cord lesions ranging from $\mathrm{C} 2$ (incomplete) to T6 volunteered to participate in this study. All subjects were successful RGO users (median use 5.7 years, range $4.1-7.3)$. Walking speed $(\mathrm{m} / \mathrm{s})$, ambulatory energy consumption $(\mathrm{J} / \mathrm{kg} / \mathrm{s})$ and energy cost $(\mathrm{J} / \mathrm{kg} / \mathrm{m})$ were derived from oxygen uptake determined using the Douglas bag technique. We anticipated that subjects walking with FES would either: (a) walk at the same speed but reduce their energy cost or (b) increase their walking speed for the same (or less) energy cost. Walking speed and energy cost remained unchanged in one subject. One subject increased his walking speed by $14.0 \%$ and increased his energy consumption by $8.4 \%$. His energy cost remained unchanged. Three subjects increased their walking speeds (by 12.4, 12.7 and 6.8\%), energy consumption (by 25.9, 20.4 and $18.4 \%$ respectively) and energy cost (by 11.6, 6.8 and $10.5 \%$ respectively). We did not find a substantial benefit, in terms of energy expenditure, from the hybrid system when walking continuously for $5 \mathrm{~min}$. We suggest that the hybrid system may be of greater benefit during prolonged walking, although other limitations, inherent in the RGO itself, may prevent an increase in compliance. Repetition of walking speed tests supported the reliability of our results. Tests on comparably aged, ablebodied subjects showed that an increase in energy cost of up to $5.8 \%$ was within the biological variability of the subject and the error of the methodology.
\end{abstract}

Keywords: energy expenditure; functional electric stimulation; gait; spinal cord lesion; reciprocating gait orthoses

\section{Introduction}

There has been much interest in the use of functional electrical stimulation (FES) for ambulation in patients with spinal cord lesions. A variety of systems have been reported ranging from FES alone ${ }^{1,2}$ to FES in combination with substantial bracing, such as the reciprocating gait orthosis $(\mathrm{RGO})^{3}$ and the hip guidance orthosis (HGO). ${ }^{4}$ One argument for combining FES with a mechanical orthosis is that the brace can contribute to, or provide, postural stability, thus allowing the FES to be used primarily to assist forward propulsion by stimulating previously passive leg muscles. In this way the problems with fatigue of stimulated postural muscles and the high energy costs involved in standing and walking with FES alone $e^{5}$ might be reduced or avoided.

Correspondence: Laura Sykes, B.S.c., North Western Orthotic Unit, Clinical Sciences Building, Hope Hospital, Eccles Old Road, Salford M6 8HD, UK
The energy requirement of standing and walking in patients with spinal cord lesions is important as it may influence the extent to which patients use walking orthoses. The present study examined a combined FES and RGO system described by Solomonow et al. $^{3}$ The RGO itself is a substantial mechanical brace which allows for a reciprocating gait pattern. Whilst energy expenditure in standing is minimal because of the postural support given by the orthosis, energy costs rise as the patient walks and uses the upper limbs and trunk to propel the body forward. A recent study found that the high energy cost of walking with the RGO alone, was due, primarily, to the great mechanical work needed to move the system, as assessed from body kinematics. ${ }^{6}$ Efforts to reduce the energy expenditure of walking with the RGO have resulted in the addition of electrical stimulation to the previously passive lower limb muscles. Using 
this large muscle mass to assist propulsion has been reported to reduce the energy requirements of walking. ${ }^{7-10}$

However, the time required to put on the FES equipment can be lengthy, particularly in addition to the RGO. The RGO itself can be time consuming and difficult to don and doff, is restrictive and in adult patients is not easily incorporated into daily activities. ${ }^{11}$ It was thought that patients would only consider such a hybrid system beneficial if it substantially reduced the energy cost of ambulation. Otherwise any advantage gained from the addition of FES might not outweigh the problems inherent in the RGO and so have no effect on patient compliance. Given that previous studies are limited, involve different methodologies, small patient numbers and show relatively small changes in energy expenditure, we thought it was important to evaluate the hybrid system on our own patients.

The purpose of the present study, therefore, was to examine the energy expenditure of five subjects using the RGO with and without FES at self selected walking speeds to assess the possible benefits of the hybrid system.

\section{Method}

\section{Subjects}

Five adult subjects (four males and one female) participated in the study. Clinical details of the subjects are outlined in Table 1. Using the ASIA impairment scale, the neurological level of the lesions ranged from $\mathrm{C} 2(\mathrm{C})$ to $\mathrm{T} 6(\mathrm{~A})$. Four subjects had suffered traumatic lesions and one (Subject 4) was diagnosed as having had a myelitic illness. Median use of the RGO was 5 years and 8 months (range 4 years 1 month to 7 years and 4 months).

\section{$R G O$}

All subjects had been fitted and trained with an RGO by a registered orthotist and physiotherapist. At the start of the study, weekly use of the RGO varied from $0.5-4 \mathrm{~h}$ by four subjects, to $25 \mathrm{~h}$ by Subject 4 .
FES

The FES protocol was derived from that used at Louisiana State University Medical Center, USA. ${ }^{3,7}$ Each subject underwent an FES programme to reverse the muscle atrophy of the quadriceps and hamstring muscle groups. The therapy consisted of three sessions per week of between 2 and 60 min each (for between 6 and 12 weeks). Stimulation was applied to the hamstrings and quadriceps muscles of each leg using conductive silicone polymer electrodes covered with karaya solid gel (Neen Pain Management Systems, UK). Neoprene thigh cuffs (Promedics, UK) held the electrodes in place. Stimulation was carried out either with the patient seated on a saddle on a hydraulic lift, which allowed the patient's legs to hang freely or in high sitting, depending on the patient's tolerance of the lift (the lift caused severe spasm in Subject 2).

Initially a low intensity stimulus was applied for two periods of 1 min each with an intervening rest period of $10 \mathrm{~min}$. In subsequent sessions the intensity of stimulus and length of treatment was gradually increased (by approximately $2 \mathrm{~min} /$ session). Stimulation automatically alternated between muscle pairings of quadriceps with contralateral hamstrings to produce a reciprocal movement of the hip(knee) into flexion and extension. When the subject could produce a strong, fatigue resistant movement for two sessions of $30 \mathrm{~min}$ with a $10 \mathrm{~min}$ intervening rest period, ankle weights of $1 \mathrm{~kg}$ were added to load the movement and improve strength. When the subject could achieve a similar fatigue resistant movement with ankle weights he/she progressed to stimulated walking.

Two stimulators were used. Four subjects used the LSU II (Louisiana State University) stimulator, which is a four channel stimulator delivering a negativegoing, monophasic, rectangular pulse at a duration of $400 \mu \mathrm{s}$, a frequency of approximately $20 \mathrm{~Hz}$ and an output range of 0 to $200 \mathrm{~mA}$. Subject 2 found the controls on this stimulator too sensitive and inadvertantly triggered his extensor spasm by using too high an intensity. This subject used a stimulator made by the Medical Physics Department, Hope Hospital, with a positive-going, monophasic, rectangular pulse of $350 \mu \mathrm{s}$ duration, a frequency of approximately $20 \mathrm{~Hz}$ and an output of 0 to $115 \mathrm{~mA}$. The controls on

Table 1 Clinical details of the subjects

\begin{tabular}{|c|c|c|c|c|c|c|c|c|}
\hline Subject & $\begin{array}{c}\text { Age } \\
\text { (years) }\end{array}$ & $\begin{array}{l}\text { Level of } \\
\text { lesion* }\end{array}$ & $\begin{array}{c}\text { Height } \\
(m)\end{array}$ & $\begin{array}{c}\text { Body } \\
\text { mass }(\mathrm{kg})\end{array}$ & $\begin{aligned} & \text { Body mass } \\
+ & \text { equipment }(\mathrm{kg})\end{aligned}$ & $\begin{array}{c}\text { Time since } \\
\text { RGO supply (years) }\end{array}$ & $\begin{array}{l}\text { Walking } \\
\text { aid }\end{array}$ & $\begin{array}{l}\text { Place of } \\
R G O \text { use }\end{array}$ \\
\hline 1 & 29 & T6 (A) & 1.80 & 64.6 & 70.8 & 5.7 & Rollator & House/garden \\
\hline 2 & 34 & T6 (B) & 1.70 & 63.4 & 69.0 & 5.5 & Rollator & House/garden \\
\hline 3 & 24 & C8 (A) & 1.85 & 72.1 & 78.0 & 4.1 & Rollator & House only \\
\hline 4 & 37 & $\mathrm{C} 2(\mathrm{C})$ & 1.55 & 96.5 & 103.6 & 7.3 & Crutches & Community \\
\hline 5 & 35 & T1 (A) & 1.83 & 84.7 & 91.7 & 7.0 & Rollator & House only \\
\hline
\end{tabular}

*Including ASIA impairment level 
this stimulator were less sensitive and the patient found them easier to use. All values assume an output impedance of $1 \mathrm{k} \Omega$.

\section{Gait training with $F E S+R G O$}

Having completed the muscle conditioning programme and having altered the subjects' rollators/crutches for use with FES, the subjects were trained to walk with the FES powered RGO (Figure 1). When deemed safe and competent with the powered system (median training period $6 \mathrm{~h}$, range $2-20$, the latter due to mechanical problems with the RGO), patients were allowed to take the FES home. The subjects were monitored regularly at review appointments when advice and training could be continued to improve skill and performance as necessary.

\section{Energy expenditure}

Energy expenditure during ambulation in the RGO was assessed without and with FES approximately 6

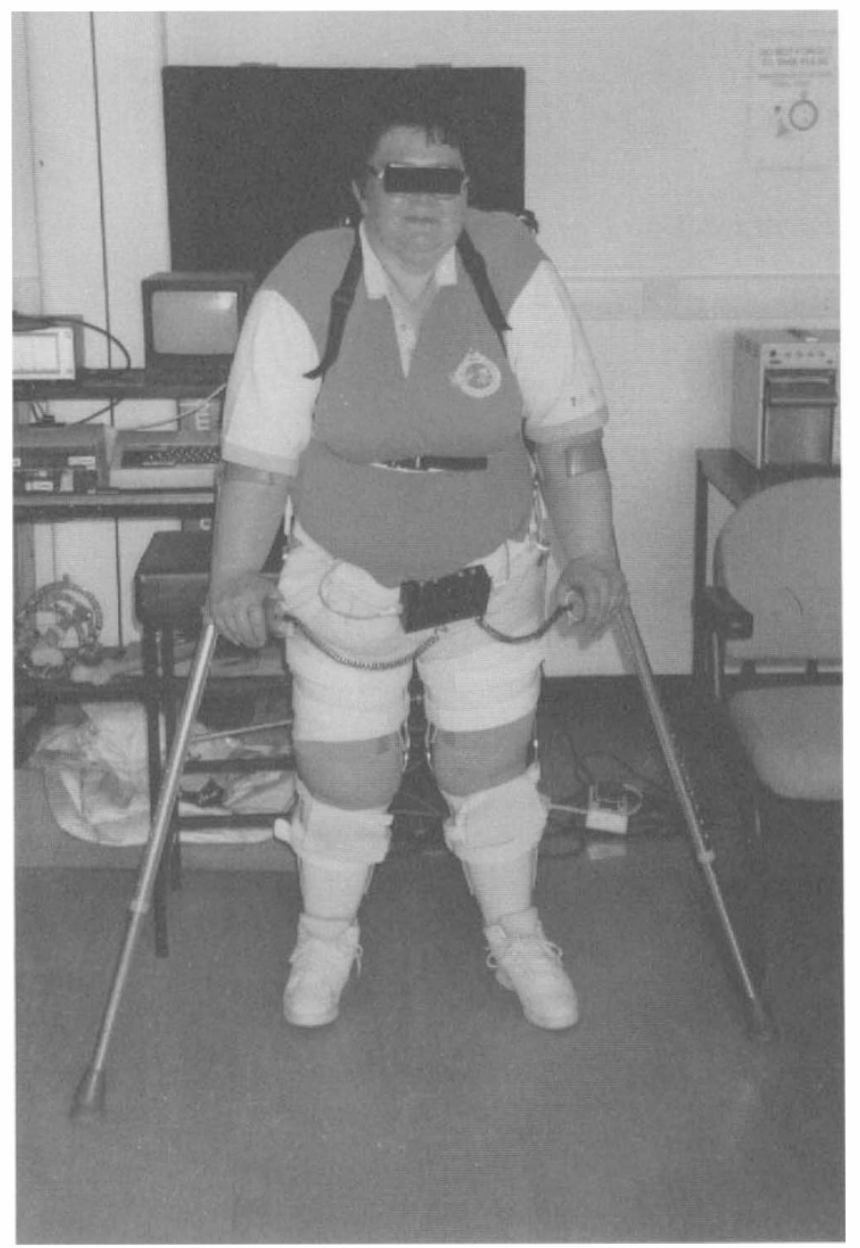

Figure 1 Subject 4 wearing the RGO and FES equipment (LSU II) months after the subject started home use of the FES system (Subject 4 was assessed after 4 months because of an imminent hospital admission). This time period allowed sufficient time for the subjects to improve their skill with the system and then to stabilise at a level of proficiency. Each subject was examined a few days before testing to ensure that the condition of the muscles had not deteriorated (i.e. that the subject could still produce the fatigue resistant performance achieved at the end of the muscle conditioning phase of the FES programme).

Oxygen consumption during ambulation was determined using the Douglas bag technique. Having familiarised the subject with the test equipment, the test track and the protocol, the subject was weighed (Seca chair). The RGO and FES equipment was weighed separately and a total weight of subject + equipment was calculated. The subject then put on the RGO and FES system. Whilst sitting at rest, expired air was collected via a lightweight mouthpiece and breathing valve connected via wide bore tubing to a 1001 Douglas bag. Following the removal of dead space, expiratory air was collected for $5 \mathrm{~min}$. Afterwards the subject walked a short distance (approximately $3 \mathrm{~m}$ ) to the start of the $20 \mathrm{~m}$ track and rested quietly, in standing, prior to the start of the test. Subjects were instructed to walk at their own preferred speed along a figure of eight track for $5 \mathrm{~min}$ using the RGO alone. Expired air was collected into a 1001 Douglas bag for the last $2 \mathrm{~min}$ of the ambulatory period. The distance covered in $5 \mathrm{~min}$ was recorded and speed calculated. Immediately after the test the subject rested quietly in the sitting position in the RGO for $1 \mathrm{~h}$. The procedure was then repeated using the FES system.

Expired air samples were analysed for fractions of oxygen and carbon dioxide (Servomex Analyser Series 1400) and evacuated (Harvard Dry Gas Meter) to determine the volume of air. Both analysers were calibrated before each series of measurements with nitrogen, a calibration gas and room air. Values were corrected for temperature, humidity and atmospheric pressure and a final value for the volume of oxygen consumed/min ( $\mathrm{min} \mathrm{VO}_{2}(\mathrm{ml} / \mathrm{min})$ ) during rest and ambulation with the RGO alone and with the hybrid walking system was calculated. From the values obtained energy consumption $(\mathrm{J} / \mathrm{kg} / \mathrm{s})$ and energy cost $(\mathrm{J} / \mathrm{kg} / \mathrm{m})$ were determined, together with walking speed. The terms adopted were those of Nene and Patrick (12) and calculations performed according to their protocol:

$$
\begin{aligned}
& \text { Energy consumption } \\
& \qquad(\mathrm{J} / \mathrm{kg} / \mathrm{s}) \\
& \frac{\text { Ambulatory min. } \mathrm{VO}_{2}(\mathrm{ml} / \mathrm{min})}{\text { Weight }(\mathrm{kg}) \times 60} \times \mathrm{K}
\end{aligned}
$$




$$
\begin{aligned}
& \begin{array}{l}
\text { Energy cost } \\
(\mathrm{J} / \mathrm{kg} / \mathrm{m})
\end{array}= \\
& \frac{\text { Ambulatory min } \mathrm{VO}_{2}(\mathrm{ml} / \mathrm{min})}{\text { Speed }(\mathrm{m} / \mathrm{min}) \times \text { weight }(\mathrm{kg})} \times \mathrm{K}
\end{aligned}
$$

where $\mathrm{K}=20.19 \mathrm{~J} / \mathrm{ml}$, since $1 \mathrm{ml} \mathrm{O}_{2}=4.825 \mathrm{cal}$ and $1 \mathrm{cal}=4.184 \mathrm{~J}$

Ambulatory energy values were calculated using the total weight moved by the subject i.e. body mass + the weight of equipment. Resting values were calculated on the basis of body mass only. All subjects were assessed with the RGO alone and then with the RGO + FES. It was thought that a rest period of one hour between tests would eliminate any effect of practice or fatigue. However, in order to support the reliability of our results walking speed was re-assessed at a later date.

\section{Reliability}

Due to the long distances travelled by the subjects to attend the laboratory for testing and the time involved, they did not repeat the test in full. Walking speed was re-assessed at a later date at a review appointment. A $40 \mathrm{~m}$ straight sided, rectangular track was employed. Subjects walked at their own preferred speed for three circuits of the track as a 'warm up', to allow for any practice effect of walking in the RGO. Allowing for intervening rest periods, when the subject's heart rate (measured by short wave telemetry using a Polar Sport Tester) returned to resting level, the walk was repeated without and then with FES. Walking speed was calculated for each of the three walks and a mean value obtained for each orthotic system.

\section{Spontaneous sources of variation}

Any measured change in the subjects may have been due to one of three things: (1) a genuine response to treatment; (2) biological variability unrelated to treatment; (3) errors in methodology. As we were unable to repeat the oxygen consumption tests on our subjects, it was thought to be important to establish the size of points (2) and (3) in an available ablebodied population. Ambulatory energy expenditure was determined in five adult subjects, tested on three occasions on the same day with at least $15 \mathrm{~min}$ rest between each test. Subjects walked at a pre-determined, comfortable walking speed which remained constant for each test. A metronome was used to maintain a constant speed. Expired air was collected as described previously during the last $2 \mathrm{~min}$ of a $5 \mathrm{~min}$ ambulatory period. Energy cost was calculated as above and the variance in values determined using ANOVA.

\section{Results}

Walking speed

Table 2 shows the walking speeds of subjects with and without electrical stimulation during the energy expenditure testing. Walking speed without FES ranged from $0.133 \mathrm{~m} / \mathrm{s}$ to $0.402 \mathrm{~m} / \mathrm{s}$ (median $=0.197$ $\mathrm{m} / \mathrm{s}$ ). With FES it ranged from $0.142 \mathrm{~m} / \mathrm{s}$ to $0.453 \mathrm{~m} / \mathrm{s}$ $($ median $=0.218 \mathrm{~m} / \mathrm{s})$. Subject 4 walked noticeably faster than the other subjects with either system. Subjects 2, 3, 4 and 5 increased their walking speed by $14.0 \%, 12.4 \%, 12.7 \%$ and $6.8 \%$ respectively when using FES. Subject 1 showed a minimal reduction in walking speed of $1 \%$ and essentially remained unchanged.

\section{Energy consumption and energy loss}

Energy consumption data is shown in Table 3. When walking with the RGO alone it ranged from $1.429 \mathrm{~J} /$ $\mathrm{kg} / \mathrm{s}$ to $3.469 \mathrm{~J} / \mathrm{kg} / \mathrm{s}$ (median $=2.071 \mathrm{~J} / \mathrm{kg} / \mathrm{s}$ ) and from $1.720 \mathrm{~J} / \mathrm{kg} / \mathrm{s}$ to $3.327 \mathrm{~J} / \mathrm{kg} / \mathrm{s} \quad$ (median $=2.588 \mathrm{~J} / \mathrm{kg} / \mathrm{s}$ ) when using FES. The four subjects who increased their walking speed with FES also increased their energy consumption. Subject 1 who had shown a minimal decrease in walking speed showed a small reduction in energy consumption of $4.1 \%$.

Energy cost (shown in Table 4) varied from $3.558 \mathrm{~J} /$ $\mathrm{kg} / \mathrm{m}$ to $17.642 \mathrm{~J} / \mathrm{kg} / \mathrm{m}$ (median $=13.519 \mathrm{~J} / \mathrm{kg} / \mathrm{m}$ ) when walking with the RGO alone and from $3.800 \mathrm{~J} / \mathrm{kg} / \mathrm{m}$ to $17.061 \mathrm{~J} / \mathrm{kg} / \mathrm{m}$ (median $=13.699 \mathrm{~J} / \mathrm{kg} / \mathrm{m}$ ) when using the hybrid system. Energy cost represents the energy required to move a unit mass over a unit distance and
Table 2 Walking speed $(\mathrm{m} / \mathrm{s})$ without and with FES measured during energy expenditure testing

\begin{tabular}{lccc}
\cline { 3 - 3 } Subject & Without FES & With FES & $\begin{array}{c}\% \text { change from } \\
\text { without } \\
\text { to with FES }\end{array}$ \\
\hline 1 & 0.197 & 0.195 & 1.0 decreased \\
2 & 0.200 & 0.228 & 14.0 increased \\
3 & 0.194 & 0.218 & 12.4 increased \\
4 & 0.402 & 0.453 & 12.7 increased \\
5 & 0.133 & 0.142 & 6.8 increased \\
\hline
\end{tabular}

Table 3 Energy consumption $(\mathrm{J} / \mathrm{kg} / \mathrm{s})$ at rest and during ambulation without and with FES

\begin{tabular}{lcccc}
\hline Subject & At rest & $\begin{array}{c}\text { Without } \\
\text { FES }\end{array}$ & $\begin{array}{c}\text { With } \\
\text { FES }\end{array}$ & $\begin{array}{c}\text { \% change from } \\
\text { without } \\
\text { to with } \text { FES }\end{array}$ \\
\hline 1 & 0.417 & 3.469 & 3.327 & 4.1 decreased \\
2 & 0.451 & 2.877 & 3.120 & 8.4 increased \\
3 & 0.490 & 2.071 & 2.588 & 25.0 increased \\
4 & 0.192 & 1.429 & 1.720 & 20.4 increased \\
5 & 0.596 & 1.798 & 2.128 & 18.4 increased \\
\hline
\end{tabular}


Table 4 Energy cost $(\mathrm{J} / \mathrm{kg} / \mathrm{m})$ of ambulation without and with FES

\begin{tabular}{lccc}
\hline & Without & $\begin{array}{l}\text { With } \\
\text { Subject }\end{array}$ & $\begin{array}{c}\text { \% change from } \\
\text { without to } \\
\text { with } F E S\end{array}$ \\
\hline 1 & 17.642 & 17.061 & 3.3 decreased \\
2 & 14.387 & 13.699 & 4.8 decreased \\
3 & 10.647 & 11.883 & 11.6 increased \\
4 & 3.558 & 3.800 & 6.8 increased \\
5 & 13.519 & 14.936 & 10.5 increased \\
\hline
\end{tabular}

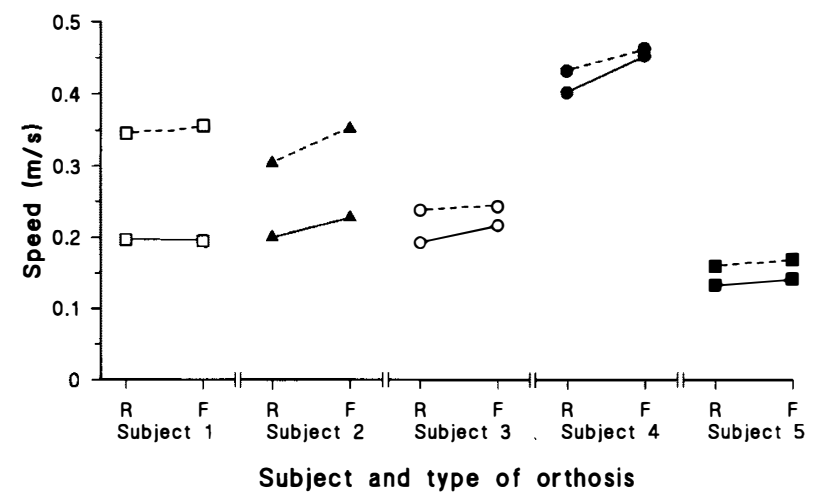

Figure 2 Walking speed of disabled subjects without (R) and with (F) FES measured during energy expenditure testing — and during a review appointment - - - -

therefore reflects the efficiency of ambulation. It can be altered by changes in speed, energy consumption or both. Subjects 1 and 2 showed a small reduction in energy cost $(3.3 \%$ and $4.8 \%$ respectively) when using the hybrid orthosis. This was calculated to be within the expected variance. Subjects 3, 4 and 5 increased their walking speeds but also increased their energy consumption by $25.0,20.0$ and $18.4 \%$ respectively. This resulted in an increased energy cost in all three subjects of 11.6, 6.8 and $10.5 \%$ respectively. Subject 4 had a particularly low energy consumption and energy cost.

\section{Reliability}

Without FES walking speeds ranged from $0.160 \mathrm{~m} / \mathrm{s}$ to $0.432 \mathrm{~m} / \mathrm{s}$ (median $=0.304 \mathrm{~m} / \mathrm{s}$ ) and with FES from $0.169 \mathrm{~m} / \mathrm{s}$ to $0.463 \mathrm{~m} / \mathrm{s} \quad($ median $=0.352 \mathrm{~m} / \mathrm{s})$. This compares with medians of $0.197 \mathrm{~m} / \mathrm{s}$ and $0.218 \mathrm{~m} / \mathrm{s}$ respectively when speed was measured during energy expenditure testing. All subjects selected a higher walking speed when using FES, though this was minimal in Subjects 1 and 3. Figure 2 shows the relationship between these walking speeds and those obtained during oxygen consumption analysis. The fact that subjects selected a higher walking speed when
Table 5 Variability in energy cost $(\mathrm{J} / \mathrm{kg} / \mathrm{m})$ in able bodied subjects

\begin{tabular}{lccccc}
\hline & & \multicolumn{3}{c}{ Energy cost $(\mathrm{J} / \mathrm{kg} / \mathrm{m})$} \\
$M / F$ & Age & & Test 1 & Test 2 & Test 3 \\
\cline { 1 - 2 } \cline { 5 - 6 } $\mathrm{F}$ & 24 & & 4.730 & 4.823 & 4.730 \\
$\mathrm{M}$ & 26 & & 4.518 & 4.393 & 4.518 \\
$\mathrm{M}$ & 30 & & 5.454 & 5.427 & 5.239 \\
$\mathrm{~F}$ & 32 & & 4.754 & 4.964 & 4.803 \\
$\mathrm{M}$ & 33 & 4.751 & 4.979 & 4.941 \\
\hline
\end{tabular}

using FES supports similar results obtained during energy expenditure testing and thus supports the reliability of our energy expenditure results. Overall walking speeds were greater during repeat testing than observed during energy expenditure testing. This is probably because the subjects were not burdened by expired air collection equipment and a larger, straight sided circuit was used.

\section{Spontaneous sources of variation}

Table 5 shows the variation in energy cost during three repeated tests in five able bodied subjects. There appears to be no order effect that could be due to fatigue or training and therefore these results are taken to reflect a combination of the variation within subjects and the measuring system. A one way ANOVA was used to determine within subjects variance. A variance of $\pm 5.8 \%$ was calculated as that value which could be expected to occur $95 \%$ of the time if two tests of energy cost were performed. Whilst this result represents an able bodied population we would expect this level of variation to occur in our disabled subject group. The percentage changes in energy cost of $3.3 \%$ and $4.8 \%$ seen in Subjects 1 and 2 respectively fell within the calculated variance.

\section{Discussion}

In the present study, efficiency of ambulation, as indicated by energy cost did not improve when using the hybrid system. Energy cost in two subjects remained unchanged. However, one of these subjects increased his walking speed. The remaining three subjects all increased their walking speed and their energy cost.

It has been stated that one major factor which will influence patients to accept or prefer one orthotic device to others is its ease of operation, which is related to the metabolic energy needed to operate it. ${ }^{7}$ In view of the physiological and physical benefits believed to be associated with standing and walking in patients with spinal cord lesions, ${ }^{13}$ it is important to encourage compliance with walking orthoses by providing the patients with the most energy efficient system available. 
On the basis of previous studies, ${ }^{7-10}$ it was anticipated that our subjects would show a reduction in $\mathrm{VO}_{2}$ and the derived energy consumption and cost when using the hybrid system. One reason why our results are not consistent with previous studies may be the method of calculation of energy expenditure. Hirokawa et $a l^{7}$ assessed ambulatory energy expenditure in 6 patients with paraplegia walking in the RGO, with or without stimulation of the thigh muscles. The authors demonstrated a reduction in energy expenditure of approximately $16 \%$ (Kcal $/ \mathrm{kg}-\mathrm{m}$ and $\mathrm{Kcal} / \mathrm{kg}$ $\mathrm{min})$ at their subjects average preferred walking speed of $0.208 \mathrm{~m} / \mathrm{s}$. Energy expenditure was calculated relative to the muscle mass believed to be working during ambulation. Energy expenditure data for the RGO alone was divided by a correction factor of 0.7 , whilst the data for the RGO+FES was not modified. This correction factor was included by the authors to compensate for the non-involvement of the lower extremity muscle mass and was based on the fact that $\mathrm{VO}_{2}$ max of arm exercise is about $70 \% \mathrm{VO}_{2} \max$ of lower limb exercise. ${ }^{14}$

In the present study there are three reasons why this calculation has not been employed. Firstly the figure of $70 \%$ relates to maximal exercise, whereas our subjects were working at submaximal levels. Secondly the figure is derived from work with able bodied subjects and not a similar disabled population. $\mathrm{VO}_{2}$ max of arm exercise may be higher in well trained paraplegics than typically reported for the able bodied population and $\mathrm{VO}_{2}$ max of leg exercise, even following training, may be lower than seen in able bodied groups. ${ }^{8}$ Thirdly, only limited leg muscles, or part thereof, will be contracting under stimulation making a correction factor of 0.7 questionable. It is interesting, however, that if our own data is modified in a manner similar to that of Hirokawa et al, ${ }^{7}$ the result would be a median decrease in energy consumption $(\mathrm{J} / \mathrm{kg} / \mathrm{s})$ of $17 \%$ (range $12-33 \%$ ) and a median decrease in energy cost $(\mathrm{J} / \mathrm{kg} / \mathrm{m})$ of $25 \%$ (range $22-33 \%$ ).

Other studies have not used such a correction factor. Petrofsky and $\mathrm{Smith}^{8}$ measured oxygen uptake (cc/min $/ \mathrm{kg} / \mathrm{m})$ in four paraplegic subjects walking in the RGO, with and without FES at a series of predetermined set walking speeds. At all speeds oxygen uptake was less when using FES than when using the RGO alone. The optimum walking speed was higher for RGO + FES ambulation and involved a lower oxygen uptake than for the RGO alone. Whilst we did not preset walking speeds, four out of five of our subjects selected a higher walking speed when using FES. However, energy cost increased in three subjects and remained unchanged in one subject.

Similarly Phillips and Hendershot ${ }^{10}$ found, in a single tetraplegic subject, that at the patient's preferred speed of $0.444 \mathrm{~m} / \mathrm{s}$, oxygen consumption $(\mathrm{ml} / \mathrm{min}$ ) was less when using the RGO + FES although this was not statistically significant.

Limited studies on small sample sizes and conflict- ing ways of dealing with data means that interpretation of our results in the light of previously published studies is difficult. Standardisation of methodology would help the interpretation of results where comparison is necessary. This is particularly important if such results are going to be used as justification for implantation programmes.

In the present study the subjects were allowed to self select their walking speed as it was thought that imposing a set walking speed would disrupt the subjects gait pattern and would not reflect their customary performance with the RGO. In our traumatic paraplegic subjects, the walking speeds seen with the RGO alone are consistent with previous studies. $^{6,7}$ It was anticipated, however, that subjects using the FES system would either walk at the same speed as with the RGO alone but reduce their energy cost, or walk faster but maintain energy costs at the same value (or less) as with the RGO alone. This was only observed in Subject 2. Four subjects demonstrated an increase in energy consumption when using FES with a resulting increase in energy cost in three subjects. An increase in energy consumption may be due to an increase in the oxygen requirements of the stimulated muscles. Indeed, other investigators have reported an increase in oxygen consumption in subjects walking at maximal speed with the RGO + FES when compared with the RGO alone. ${ }^{15}$ It is interesting that in the case of three subjects in the present study, there was some factor which influenced them to walk faster despite increasing their energy consumption and energy cost. One possibility for this was that there was an ordering effect which was carried over the one hour rest period between tests. This would seem unlikely as all subjects were experienced RGO users. In addition, repeat speed testing, allowing for a practice effect, supported the results obtained during energy expenditure measurements.

A second possibility is the physiological level of effort involved in using the arms alone to operate the RGO. Physiological responses to arm work (ie heart rate, lactate levels, blood pressure and ventilation) are similar to, but greater than, the responses to equivalent leg work. ${ }^{16,17}$ Other studies have shown that heart rate ran $^{7,9}$ and lactate ${ }^{15}$ are reduced when FES is used in RGO ambulation, suggesting that the FES system may be beneficial in reducing the strain from upper limb work. Electromyogram studies have also shown that the activity in upper body muscles was much higher when walking in RGOs without FES than in RGOs with FES. ${ }^{8}$ Physiologically, therefore, a small increase in overall energy cost may be acceptable to the subject who benefits from increased walking speed, a reduction in physiological strain and a real reduction in stress on the arms. This is supported by comments from the subjects, for example that the FES system slightly reduces the effort of walking and is less tiring on the back of the neck and the shoulders.

The fact that four subjects selected a higher walking 
speed over 5 min when using FES also suggests that they may be able to maintain a faster speed for longer with this system, suggesting that fatigue is less of a problem when the RGO and FES are employed together. This may be due to the fact that venous return has been shown to be enhanced during $\mathrm{FES}^{18}$ which may have the effect of aiding cardiovascular control, improving the delivery of oxygen to the working muscles and thus reducing the build up of lactic acid and the onset of fatigue. ${ }^{18}$ However, this may only be useful if the RGO is used over long distances. As shown in Table 1, four of the subjects use the RGO around the home only. This is not solely because of the effort required to walk, but because of other limitations it imposes. The wheelchair provides a more functional system outside of the home, when it allows for greater mobility, manoeuvrability, function, safety and speed. Any advantage gained from the FES augmented RGO may therefore be lost in the home environment when walking distances are small. A possible positive effect of the FES system on patient compliance is therefore questionable and requires further investigation.

\section{Acknowledgements}

This work was supported by a Medical Innovation Grant from the North West Region of the NHS. Our thanks to Professor M Solomonow of Louisiana State University for help with the stimulation protocol and Professor P Bowker of the School of Prosthetics and Orthotics, Salford for contributing facilties for the study.

\section{References}

1 Marsolais EB, Kobetic R. Development of a practical electrical stimulation system for restoring gait in the paralysed patient. Clin Orthop Rel Res 1988; 233: 64-74.

2 Malezic M, Hesse S. Restoration of gait by functional electrical stimulation in paraplegic patients: a modified programme of treatment. Paraplegia 1995; 33: 126-131.
3 Solomonow M et al. The RGO generation II: muscle stimulation powered orthosis as a practical walking system for thoracic paraplegics. Orthopedics 1989; 12: 1309-1315.

4 McClelland $\mathrm{M}$ et al. Augmentation of the Oswestry Parawalker orthosis by means of surface electrical stimulation: gait analysis of three patients. Paraplegia 1987; 25: $32-38$.

5 Marsolais EB, Edwards BG. Energy costs of walking and standing with functional neuromuscular stimulation and long leg braces. Arch Phys Med Rehabil 1988; 69: 243-249.

6 Bernardi $\mathrm{M}$ et al. The efficiency of walking of paraplegic patients using a reciprocating gait orthosis. Paraplegia 1995; 33: 409415.

7 Hirokawa $\mathrm{S}$ et al. Energy consumption in paraplegic ambulation using the reciprocating gait orthosis and electrical stimulation of the thigh muscles. Arch Phys Med Rehabil 1990; 71: 687-694.

8 Petrofsky JS, Smith JB. Physiologic costs of computer-controlled walking in persons with paraplegia using a reciprocating-gait orthosis. Arch Phys Med Rehabil 1991; 72: 890-896.

9 Isakov E, Douglas R, Berns P. Ambulation using the reciprocating gait orthosis and functional electrical stimulation. Paraplegia 1992; 30: 239-245.

10 Phillips CA, Hendershot DM. Functional electrical stimulation and reciprocating gait orthosis for ambulation exercise in a tetraplegic patient: a case study. Paraplegia 1991; 29: 268-276.

11 Sykes L, Edwards J, Powell ES, Ross ERS. The reciprocating gait orthosis (RGO): long term usage patterns. Arch Phys Med Rehabil 1995; 76: 779-783.

12 Nene AV, Patrick JH. Energy cost of paraplegic locomotion using the Parawalker-electrical stimulation 'hybrid' orthosis. Arch Phys Med Rehabil 1990; 71: 116-120.

13 Bromley I. Tetraplegia and paraplegia: a guide for physiotherapists. 3rd ed. Churchill Livingstone, Edinburgh, 1985.

14 Astrand P, Rodahl K. Textbook of work physiology. 2nd ed. McGraw-Hill, New York, 1977.

15 Thoumie $\mathrm{P}$ et al. Restoration of functional gait in paraplegic patients with the RGO-II hybrid orthosis. A multicentre controlled study. I. Clinical evaluation. Paraplegia 1995; 33: $647-653$.

16 Stenberg J et al. Hemodynamic response to work with different muscle groups, sitting and supine. J Appl Physiol 1967; 22: 61 70 .

17 Bevegard S, Freychuss U, Standell J. Circulatory adaptation to arm and leg exercise in supine and sitting position. J Appl Physiol 1966; 21: $37-46$

18 Davis GM et al. Cardiovascular responses to arm cranking and FNS-induced leg exercise in paraplegics. Am Phys Soc 1990; 69: $672-677$. 